\title{
Geometric Nonlinear Analysis for Frame Structures, Part 2: Dynamic
}

\author{
Analysis \\ Duan Jin ${ }^{1, a}$, Li Yun-gui ${ }^{1}$ \\ ${ }^{1}$ China State Construction Technical Center, Beijing, 101300, PR China \\ a duanjin78@126.com
}

\begin{abstract}
Key words: structural simulation, system integration, finite element analysis
Abstract: In this paper, the governing equation of dynamic analysis for frame structures is derived, with geometric nonlinearity considered. In the process of derivation, the updated Lagrange format is adopted and the elemental equilibrium equation is established at current configuration and then transformed into the initial configuration. To simplify the calculation of mass matrix, it is assumed that the beam's mass is only related with its rigid configuration. In other words, the mass effect due to the beam's deformation is ignored. Finally, a numerical example about dynamic buckling analysis of a circular arch subjected to a uniformly distributed step load is presented and discussed detailedly, demonstrating the validity and accuracy of the present method.
\end{abstract}

\section{Introduction}

The geometric nonlinear analysis of beam-structures is a very classical problem, which has attracted many researchers in the past half century. Since it is not a simple extension from its counterpart of linear analysis, many researchers have paid their attention on this field. Bathe and Bolourchi [1] presented a Total Lagrange (TL) formulation and an Updated Lagrange (UL) formulation for large rotation nonlinear analysis of three-dimensional beams. Belyschko and Hsieh [2] proposed a nonlinear transient Finite Element (FE) analysis for two-dimensional beams with convected co-ordinates. Yang, Kuo and $\mathrm{Wu}$ [3] derived a co-rotational formulation for structural frames, in which the displacements of each element were subdivided into rigid body displacements and natural deformations.

In this paper, the governing equation of dynamic analysis for frame structures is derived, with geometric nonlinearity considered. In the process of derivation, the updated Lagrange format is adopted and the elemental equilibrium equation is established at current configuration and then transformed into the initial configuration. To simplify the calculation of mass matrix, it is assumed that the beam's mass is only related with its rigid configuration. In other words, the mass effect due to the beam's deformation is ignored. Finally, a numerical example about dynamic buckling analysis of a circular arch subjected to a uniformly distributed step load is presented and discussed detailedly, demonstrating the validity and accuracy of the present method.

\section{The governing equation of dynamic analysis considering geometric nonlinearity}

The derivation of dynamic government equation is similar with that of static government equation. The updated Lagrange format is adopted and the elemental equilibrium equation is established at current configuration and transformed into the initial configuration, see Figure 1.

From virtual work principle it can be concluded that 


$$
\int_{0}^{l} \iint_{A}\left[{ }_{t}^{t+\Delta t} \sigma_{x x} \delta_{t} \varepsilon_{x x}+{ }^{t+\Delta t}{ }_{t} \sigma_{x y} \delta_{t} \gamma_{x y}+{ }^{t+\Delta t}{ }_{t} \sigma_{x z} \delta_{t} \gamma_{x z}\right] d y d z d x={ }^{t+\Delta t} \mathfrak{A}
$$

Where ${ }^{t+\Delta t} \mathfrak{A}$ denotes the virtual work by external force and inertia force, which is essentially a kind of body force and can be stated as the following equation

$$
{ }^{t+\Delta t} \mathfrak{A}=\cdots-\int_{0}^{l} \iint_{A} \rho^{t+\Delta t}{ }_{t} \ddot{u}_{k} \delta_{t} u_{k} d A d l
$$

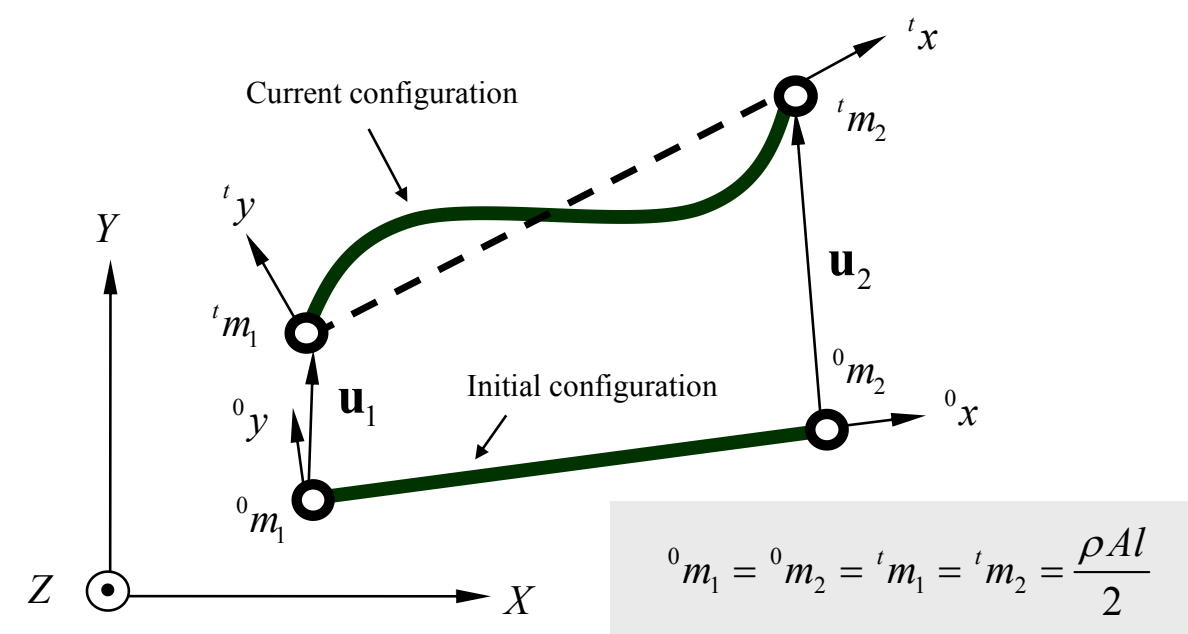

Figure 1 the configuration of beam element in motion

To simplify the calculation of inertia force, it is assumed that the beam's mass is only related with its rigid configuration. In other words, the mass effect resulted from its deformation is not taken into account. Shown in Figure 1, the original length of beam is denoted as $l$, density as $\rho$, cross-section area as $A$, torsional moment as $J$, so the concentrated mass and mass moment at the beam end-points is $\rho A l / 2$ and $\rho J l / 2$ respectively. Equation (2) can be stated as

$$
{ }^{t+\Delta t} \mathfrak{A}=\cdots-\frac{\rho A l}{2} \sum_{i=1}^{2}\left({ }^{t+\Delta t} \ddot{u}_{i} \delta_{t} u_{i}+{ }^{t+\Delta t} \ddot{t}_{i} \delta_{t} v_{i}+{ }^{t+\Delta t} \ddot{w}_{i} \delta_{t} w_{i}\right)-\frac{\rho J l}{2} \sum_{i}^{2}\left({ }^{t+\Delta t} \ddot{\theta}_{x i} \delta_{t} \theta_{x i}\right)
$$

Where $i=1,2$ denotes node number, ${ }_{t} u_{i} 、{ }_{t} v_{i} 、{ }_{t} w_{i}$ and ${ }_{t} \theta_{x i}$ denotes the nodal displacement increment from time $t$ to $t+\Delta t,{ }^{t+\Delta t} \ddot{u}_{i} 、{ }^{t+\Delta t} \ddot{v}_{i} 、{ }^{t+\Delta t} \ddot{w}_{i}$ and ${ }^{t+\Delta t} \ddot{\theta}_{x i}$ denotes the nodal acceleration at time $t+\Delta t$. All of the above variates are measured in the configuration at time $t$, see Figure 1 .

Equation (3) can be expressed as the matrix form

$$
{ }^{t+\Delta t} \mathfrak{A}=\cdots-\delta\left({ }_{t} \mathbf{a}^{e}\right)^{T}{ }_{t} \mathbf{M}^{t+\Delta t}{ }_{t} \ddot{\mathbf{a}}^{e}
$$

Where ${ }_{t} \mathbf{M}$ is the elemental concentrated mass matrix in the configuration at time $t,{ }_{t} \mathbf{a}^{e}$ is the 
nodal general displacement increment vector, see Equation (5), ${ }^{t+\Delta t}{ }_{t} \ddot{\mathbf{a}}^{e}$ is the nodal general acceleration vector at time $t+\Delta t$

$$
{ }_{t} \mathbf{a}^{e}=\left\{\begin{array}{ll}
\mathbf{a}_{1}^{e} & { }_{t} \mathbf{a}_{2}^{e}
\end{array}\right\}^{\mathrm{T}}
$$

Substituting Equation (4) into (1) and using the traditional derivation, the following equation can be obtained

$$
{ }_{t} \mathbf{M}_{t} \ddot{\mathbf{a}}^{e}+\left({ }_{t}^{t} \mathbf{K}_{L}+{ }_{t}^{t} \mathbf{K}_{G}\right){ }_{t} \mathbf{a}^{e}=\left({ }^{t+\Delta}{ }_{t} \mathbf{P}_{e}-{ }_{t}^{t} \mathbf{P}\right)
$$

Where ${ }_{t} \mathbf{M}$ is the elemental concentrated mass matrix, ${ }_{t}^{t} \mathbf{K}_{L}$ is the linear stiffness matrix, ${ }_{t}^{t} \mathbf{K}_{G}$ is the geometrical nonlinear stiffness matrix, and ${ }_{t}^{t} \mathbf{P}$ is the nodal internal force vector

\section{Numerical example}

The problem considered here is a dynamic buckling analysis of a circular arch subjected to a uniformly distributed step load (see Figure 2). In the analysis, the ratio of time step over fundamental period $\Delta t / T_{f}$ is approximately $1 / 70$, which is identical with the ratio adopted by Bathe et al. [4]. Figure 3 compares the displacement response predicted in the present study with the results of Bathe et al. [4], Humphreys [5], Noor and Knight [6]. In that figure, the deflection ratio $\Delta$, the time parameter $\tau$, and the load parameter $P$ are defined as:

$$
\Delta=\frac{w}{H / 2} \quad ; \quad \tau=\sqrt{\frac{E}{\rho}} \times \frac{t}{R} \quad ; \quad P=\left(\frac{R}{h}\right)^{2} \times \frac{q}{E}
$$

where $w$ is the average normal deflection at arch top.

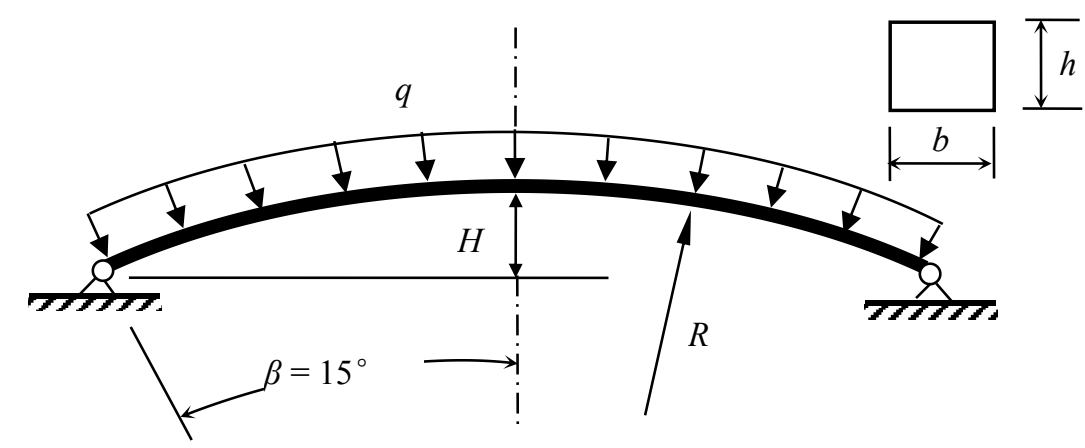

$$
\begin{array}{ll}
R=170.47 \mathrm{~cm} & E=6.90 \times 10^{10} \mathrm{~Pa} \\
b=h=2.54 \mathrm{~cm} & v=0.20 \\
\rho=2.61 \times 10^{3} \mathrm{~kg} / \mathrm{m}^{3} & H=5.81 \mathrm{~cm} \\
P=(R / h)^{2} \times(q / E) &
\end{array}
$$

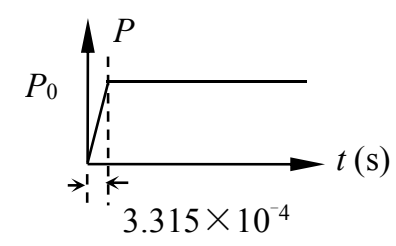

Figure 2 A simply supported shallow arch subjected to a uniformly distributed step load 
In Ref. [4], the dynamic snap-through buckling of the arch is described as a relatively large increase in deflection over a relatively short period of time and a noticeable change in the equilibrium position. The present results are compared with those in literatures in loading cases of $P=0.205$ and $P=0.25$ in Fig. 14, from which one can conclude that the critical buckling load predicted by the present method lies between $P=0.195$ and $P=0.205$, which is slightly lower than $P=0.210 \sim 0.215$ reported by Humphreys [5], while slightly higher than $P=0.190 \sim 0.200$ reported by Bathe et al. [4]. Moreover, as observed by Humphreys [5] that: once the arch has popped through, it just oscillates about an equilibrium position. Further increases in loading do not appreciably change the maximum deflection, but just simply accelerate the buckling occurrence.

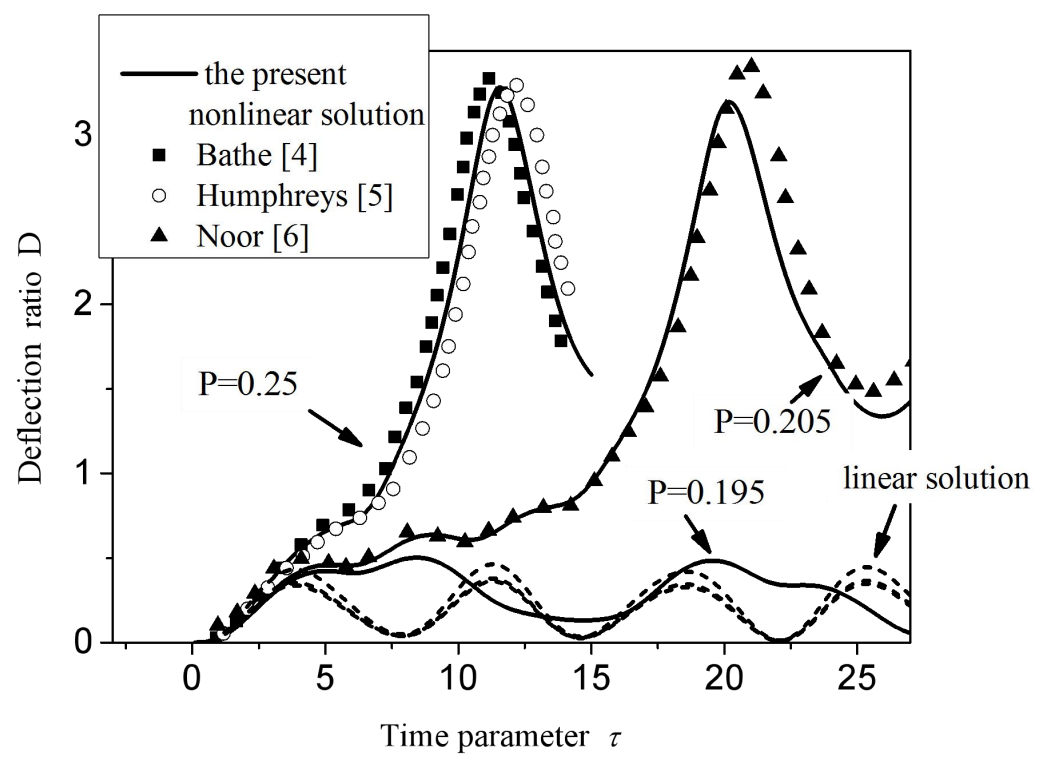

Figure 3 Dynamic snap-through of a shallow arch

\section{Summary}

In this paper, the governing equation of dynamic analysis for frame structures is derived, with geometric nonlinearity considered. In the process of derivation, the updated Lagrange format is adopted and the elemental equilibrium equation is established at current configuration and then transformed into the initial configuration. To simplify the calculation of mass matrix, it is assumed that the beam's mass is only related with its rigid configuration. In other words, the mass effect due to the beam's deformation is ignored. Finally, a numerical example about dynamic buckling analysis of a circular arch subjected to a uniformly distributed step load is presented and discussed detailedly, demonstrating the validity and accuracy of the present method.

\section{Reference}

[1] K.J. Bathe and S. Bolourchi, Large Displacement Analysis of Three-dimensional Beam Structures. International Journal for Numerical Methods in Engineering, vol. 14, pp. 961-986, 1979.

[2] T. Belytschko and J. Hsieh, Non-linear Transient Finite Element Analysis with Convected 
Co-ordinates, International Journal for Numerical Methods in Engineering, vol. 7, pp. 255-271, 1973.

[3] Y.B. Yang, S.R. Kuo and YS. Wu, Incrementally Small-Deformation Theory for Nonlinear Analysis of Structural Frames, Engineering Structures, vol. 24: 783-798, 2002.

[4] Bathe K J, Ramm E, Wilson E L. Finite Element Formulations for Large Deformation Dynamic Analysis. International Journal for Numerical Methods in Engineering, 1975, 9: 353-386

[5] Humphreys J S. On Dynamic Snap Buckling of Shallow Arches. AIAA J., 1966, 4: 878-886

[6] Noor A K, Knight N F. Nonlinear Dynamic Analysis of Curved Beams. Computer Methods in Applied Mechanics and Engineering, 1980, 23: 225-251 